Applied Mathematical Sciences, Vol. 9, 2015, no. 52, 2567 - 2577

HIKARI Ltd, www.m-hikari.com

http://dx.doi.org/10.12988/ams.2015.52125

\title{
Application of Block Matrix Theory to Obtain the Inverse Transform of the Vector-Valued DFT
}

\author{
Pablo Soto-Quiros \\ Centre for Industrial and Applied Mathematics \\ University of South Australia \\ SA 5095, Australia \\ and \\ Escuela de Matemáticas \\ Instituto Tecnológico de Costa Rica \\ Apdo. 159-7050, Cartago, Costa Rica
}

Copyright (c) 2015 Pablo Soto-Quiros. This is an open access article distributed under the Creative Commons Attribution License, which permits unrestricted use, distribution, and reproduction in any medium, provided the original work is properly cited.

\begin{abstract}
We study the vector-valued discrete Fourier transform (vector-valued DFT) and its inverse transform, the vector-valued DFT inversion, through its block matrix representation. These transforms are defined for $\mathrm{N}$ periodic signals, taking values in $\mathbb{C}^{D}$. The vector-valued DFT is a generalization of classical discrete Fourier transform definition. The goal of this paper is present necessary and sufficient new conditions to vectorvalued DFT inversion, and so, to make a perfect recovery of original signal.
\end{abstract}

Mathematics Subject Classification: 15A99

Keywords: DFT matrix, vector-valued DFT, vector-valued DFT inversion, DFT frames

\section{Introduction}

The ambiguity function is useful for describing the behavior of continuous radar signals. This function plays a central role in both the detection and the resolu- 
tion theory for moving targets $[5,7,8]$. A computational implementation of the ambiguity function is performed using discrete periodic signals and the discrete ambiguity function $[1,6]$. A signal is a discrete periodic signal if it completes a pattern within a measurable time frame, called a period and repeats that pattern over identical subsequent periods. The construction of new complex valued constant amplitude zero autocorrelation (CAZAC) discrete periodic signals [1], which serve as coefficients for phase coded waveforms with prescribed discrete ambiguity function behavior, was the motivation for Benedetto and Donatelli [2] to extend the discrete ambiguity function concept to $N$-periodic discrete periodic signals $x$, taking values in $\mathbb{C}^{D}$. This new function is called the vector-valued ambiguity function. The vector-valued ambiguity function is defined for the study of vector-valued CAZACs, which are relevant in light of vector sensor and MIMO technologies [2]. This study is located in the area of digital signals processing.

In order to develop the definition of vector-valued ambiguity function, Benedetto and Donatelli also define a vector-valued discrete Fourier transform (vector-valued DFT). The vector-valued DFT is defined for $N$-periodic signal $x$, taking values in $\mathbb{C}^{D}$, i.e., the vector-valued DFT is the map $\mathcal{F}_{x}^{\mathcal{A}}: \mathbb{Z}_{N} \rightarrow \mathbb{C}^{D}$ such that

$$
\mathcal{F}_{x}^{\mathcal{A}}(k)=\frac{1}{\sqrt{D}} \sum_{n \in \mathbb{Z}_{N}} x_{n} \odot e_{N}^{-n k}
$$

where each $x_{n}$ and $e_{N}^{-n k}$ are vectors of length $D, \odot$ is the Hadamard product and $\mathcal{A} \subseteq\{0,1, \ldots, N-1\}$. In [2] also defines a vector-valued DFT inversion, but not specify under what conditions the vector-valued DFT inversion is well defined, and so, recover the original signal $x$.

This paper presents necessary and sufficient new conditions to the vectorvalued DFT inversion. This study is performed through the block matrix representation of vector-valued DFT, where each block is a complex vector. Also, we explain and develops some concepts related to the block matrix, in addition to defining some operations, operators and new results.

The paper is organized as follows. In Section 2, we explain the notation, some definitions and auxiliary results. In Section 3, we define some results for the block matrix. In Section 4, we explain vector-valued DFT and its inverse transform, specifying under what conditions the vector-valued DFT inversion is well defined. Finally, in Section 5, we present some conclusions. 


\section{Background}

\subsection{Notation}

Let $\mathbb{Z}_{N}=\{0,1,2, \ldots, N-1\}$ be the additive group $\mathbb{Z}$ of integers module $N$, $\mathbb{C}^{M \times N}$ the matrix space of $M$ rows and $N$ columns, whose entries are complex numbers. Special case is $\mathbb{C}^{N \times 1}=\mathbb{C}^{N}$ : the complex vector space of size $N$. As a signal $x$ can be represented by elements in $\mathbb{C}^{N}$, then $x \in \mathbb{C}^{N}$. For the purposes of this paper, the rows and columns of any matrix $\boldsymbol{A} \in \mathbb{C}^{M \times N}$ are indexed by elements of $\mathbb{Z}_{M}$ and $\mathbb{Z}_{N}$, respectively. $\boldsymbol{A}(m, n)$ represents the entry $(m, n)$ of $\boldsymbol{A}$, for $(m, n) \in \mathbb{Z}_{M} \times \mathbb{Z}_{N} . \quad \boldsymbol{I}_{N} \in \mathbb{C}^{N \times N}$ is the identity matrix of order $N$. $\boldsymbol{U}_{M, N} \in \mathbb{C}^{M \times N}$ is the matrix of order $M \times N$ whose entries are equals to 1 , and $\boldsymbol{U}_{M, N}=\boldsymbol{U}_{N}$ if $M=N$. The Hadamard product (or pointwise multiplication) of $\boldsymbol{A}, \boldsymbol{B} \in \mathbb{C}^{M \times N}$ is defined as $\boldsymbol{A} \odot \boldsymbol{B} \in \mathbb{C}^{M \times N}$ such that $(\boldsymbol{A} \odot \boldsymbol{B})(m, n)=\boldsymbol{A}(m, n) \cdot \boldsymbol{B}(m, n)$. Also, the Hadamard product of set $\left\{\boldsymbol{A}_{d}\right\}_{d \in \mathbb{Z}_{D}} \subset \mathbb{C}^{M \times N}$ is defined as

$$
\boldsymbol{A}=\bigodot_{d \in \mathbb{Z}_{D}} \boldsymbol{A}_{d}=\boldsymbol{A}_{0} \odot \boldsymbol{A}_{1} \odot \ldots \odot \boldsymbol{A}_{D-1},
$$

where $\boldsymbol{A} \in \mathbb{C}^{M \times N}$. As $\mathbb{Z}_{0}$ is not a defined set, then, for our purposes, when $D=0$, equation (1) is defined as:

$$
\bigodot_{d \in \mathbb{Z}_{0}} \boldsymbol{A}_{d}=\boldsymbol{U}_{M, N}
$$

\subsection{Discrete Fourier Transform}

Let $x \in \mathbb{C}^{N}$. The discrete Fourier transform (DFT) of order $N$ of $x$ is defined as the map $\mathcal{F}_{x}: \mathbb{Z}_{N} \rightarrow \mathbb{C}$ such that $\mathcal{F}_{x}(k)=\sum_{n \in \mathbb{Z}_{N}} x(n) \omega_{N}^{-n k}$, with $\omega_{N}=e^{2 \pi i / N}$. The key properties of the DFT are based on the following elementary identity

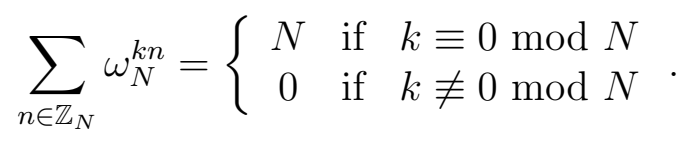

The DFT matrix of order $N$, denoted by $\boldsymbol{F}_{N} \in \mathbb{C}^{N \times N}$, is defined as $\boldsymbol{F}_{N}(m, n)=\omega_{N}^{-m n}$. For $x \in \mathbb{C}^{N}$, the matrix representation of DFT of $x$ is $\mathcal{F}_{x}=\boldsymbol{F}_{N} x$. The following result seems quite intuitive (as noted in [9] and [4]), but we did not find anywhere in the literature a formal statement and proof for it. So, for the sake of completeness we decided to include it here.

Lemma 1. Let $D \in \mathbb{Z}_{N} \backslash\{0\}$ and $\boldsymbol{F} \in \mathbb{C}^{N \times N}$ such that

$$
\boldsymbol{F}=\bigodot_{d \in \mathbb{Z}_{D}} \boldsymbol{F}_{N}=\underbrace{\boldsymbol{F}_{N} \odot \boldsymbol{F}_{N} \odot \ldots \odot \boldsymbol{F}_{N}}_{D \text { times }} .
$$

Then: 
1. if $N$ is composite, then exist $D \in \mathbb{Z}_{N} \backslash\{0\}$ such that $\boldsymbol{F}$ is not invertible.

2. if $N$ if prime, then $\boldsymbol{F}$ is invertible for all $D \in \mathbb{Z}_{N} \backslash\{0\}$.

Proof. The matrix $\boldsymbol{F}$ can be expressed as a Vandermonde matrix:

$$
\boldsymbol{F}=\left(\begin{array}{ccccc}
1 & 1 & 1 & \cdots & 1 \\
a_{0} & a_{1} & a_{2} & \cdots & a_{N-1} \\
a_{0}^{2} & a_{1}^{2} & a_{2}^{2} & \cdots & a_{N-1}^{2} \\
\vdots & \vdots & \vdots & \ddots & \vdots \\
a_{0}^{N-1} & a_{1}^{N-1} & a_{2}^{N-1} & \cdots & a_{N-1}^{N-1}
\end{array}\right)=V\left(a_{0}, a_{1}, a_{2}, \ldots, a_{N-1}\right)
$$

where $a_{n}=\omega_{N}^{-n D}$, for $n \in \mathbb{Z}_{N}$. The matrix $\boldsymbol{F}$ is invertible if and only if all $a_{n}$ are distincts [9].

1. If $N$ is a composite number, then exist two positive integer numbers $R, S, R<N$ and $S<N$, such that $N=R S$. Choose $D=R$. Then, $a_{0}=1$ and $a_{S}=\omega_{N}^{-S R}=\omega_{N}^{-N}=1$. As $a_{0}=a_{S}$, thus it follows that $\boldsymbol{F}$ is not invertible.

2. By contradiction. Assume that exist $m, n \in \mathbb{Z}_{N}, m \neq n$, such that $a_{m}=a_{n}$. Without loss of generality, $n<m$. $N$ is prime, $0<m-n<N$ and $D<N$, then, g.c.d. $(N, m-n)=1$ and g.c.d. $(N, D)=1$, giving g.c.d. $(N,(m-n) D)=1$. This implies

$$
N \nmid(m-n) D .
$$

Now, $a_{m}=a_{n}$ is the same that $\omega_{N}^{-m D}=\omega_{N}^{-n D}$, then $m D \equiv n D \bmod N$, i.e., $N \mid(m-n) D$, which contradicts the statement (3). Then, $a_{m} \neq a_{n}$, for all $m \neq n$. Thus, it follows that $\boldsymbol{F}$ is invertible for all $D \in \mathbb{Z}_{N} \backslash\{0\}$.

\subsection{DFT Frames}

A set $\mathcal{G}=\left\{f_{n}\right\}_{n \in \mathbb{Z}_{N}} \subset \mathbb{C}^{D}$ is a finite frames for $\mathbb{C}^{D}$ if exists two positive real numbers $a, b$ such that for all $x \in \mathbb{C}^{D}$

$$
a\|x\|^{2} \leq \sum_{n \in \mathbb{Z}_{N}}\left|\left\langle x, f_{n}\right\rangle\right|^{2} \leq b\|x\|^{2} .
$$

A frame $\mathcal{G}$ is a tight frame if exist $a=b$. In addition, if each $f_{n}$ is unitnorm, then $\mathcal{G}$ is a finite unit-norm tight frame. A finite unit norm tight frame is referred as a FUNTF. Let two positive integer numbers $N$ and $D, N \geq$ $D$. The DFT frames in $\mathbb{C}^{D}$ is the set $\left\{1 / \sqrt{D} \cdot e_{N}^{n}\right\}_{n \in \mathbb{Z}_{N}}$ such that $e_{N}^{n}=$ $\left(\omega_{N}^{n \alpha_{1}}, \omega_{N}^{n \alpha_{2}}, \ldots, \omega_{N}^{n \alpha_{D}}\right)^{T}$, where $\mathcal{A}=\left\{\alpha_{1}, \alpha_{2}, \ldots, \alpha_{D}\right\} \subseteq \mathbb{Z}_{N}$, with $\alpha_{j}<\alpha_{k}$ if and only if $j<k$. The DFT frames are a FUNTF [3]. For a fixed value $N$, $\mathbb{C}^{D}$ has $\left(\begin{array}{l}N \\ D\end{array}\right)=\frac{N !}{D !(N-D) !}$ DFT frames sets, because each DFT frame depends on the elements of $\mathcal{A}$. 


\section{Block Matrices}

A block matrix $\boldsymbol{A} \in \mathbb{C}^{P \times Q}$ with $M$ row partitions and $N$ column partitions is defined as

$$
\boldsymbol{A}=\left(\begin{array}{ccc}
\boldsymbol{A}_{0,0} & \cdots & \boldsymbol{A}_{0, N-1} \\
\vdots & \ddots & \vdots \\
\boldsymbol{A}_{M-1,0} & \cdots & \boldsymbol{A}_{M-1, N-1}
\end{array}\right)
$$

where $\boldsymbol{A}_{m, n}$ designates the $(m, n)$ block [9]. In this paper, we use block matrices where each block is a complex vector of size $D$, i.e., $\boldsymbol{A} \in \mathbb{C}^{D M \times N}$ and $\boldsymbol{A}_{m, n} \in$ $\mathbb{C}^{D}$.

The block matrix $\boldsymbol{A}$ can be defined from an indexed and unique set of $D$ matrices in $\mathbb{C}^{M \times N}:\left\{\boldsymbol{A}_{d}\right\}_{d \in \mathbb{Z}_{D}} \subset \mathbb{C}^{M \times N}$ and this set is called generator set. We define the generator operator as the map $\bigvee: \prod_{d \in \mathbb{Z}_{D}} \mathbb{C}^{M \times N} \rightarrow \mathbb{C}^{D M \times N}$ such that

$$
\boldsymbol{A}=\bigvee_{d \in \mathbb{Z}_{D}} \boldsymbol{A}_{d}
$$

where $\boldsymbol{A}_{m, n}=\left(\boldsymbol{A}_{0}(m, n), \boldsymbol{A}_{1}(m, n), \ldots, \boldsymbol{A}_{D-1}(m, n)\right)^{T}$. Let $\left\{\boldsymbol{A}_{d}\right\}_{d \in \mathbb{Z}_{D}} \subset \mathbb{C}^{M \times N}$ and $\left\{\boldsymbol{B}_{d}\right\}_{d \in \mathbb{Z}_{D}} \subset \mathbb{C}^{M \times N}$ the generator sets of $\boldsymbol{A}, \boldsymbol{B} \in \mathbb{C}^{D M \times N}$, respectively. Some operations for block matrices are also defined from the generator set and generator operator:

- Addition: $\boldsymbol{A}+\boldsymbol{B}=\bigvee_{d \in \mathbb{Z}_{D}}\left(\boldsymbol{A}_{d}+\boldsymbol{B}_{d}\right)$,

- Scalar Product: For $\beta \in \mathbb{C}, \beta \boldsymbol{A}=\bigvee_{d \in \mathbb{Z}_{D}} \beta \boldsymbol{A}_{d}$,

- Transpose: $\boldsymbol{A}^{T}=\bigvee_{d \in \mathbb{Z}_{D}} \boldsymbol{A}_{d}^{T}$ and

- Conjugate: $\overline{\boldsymbol{A}}=\bigvee_{d \in \mathbb{Z}_{D}} \overline{\boldsymbol{A}_{d}}$.

Let $\boldsymbol{A} \in \mathbb{C}^{D M \times N}$ and $\boldsymbol{C} \in \mathbb{C}^{D N \times P}$ two block matrices. For the purposes of this paper, we define the operation $\boldsymbol{A} * \boldsymbol{C} \in \mathbb{C}^{D M \times P}$ such that

$$
(\boldsymbol{A} * \boldsymbol{C})_{m, q}=\sum_{n \in \mathbb{Z}_{N}} \boldsymbol{A}_{m, n} \odot \boldsymbol{C}_{n, q} .
$$

The $\boldsymbol{A} * \boldsymbol{C}$ operation can be performed if $\boldsymbol{A}$ and $\boldsymbol{C}$ have same complex vector block size, the size of each block divides the number of rows of both matrices and the number of rows of $C$ divided by the number of columns of $A$ is equal to the size of each block. Also $\boldsymbol{A} * \boldsymbol{C}=\bigvee_{d \in \mathbb{Z}_{D}} \boldsymbol{A}_{d} \boldsymbol{C}_{d}$. 
With operation defined above, $\left(\mathbb{C}^{D N \times N},+, \cdot, *\right)$ is a associative algebra over the field $\mathbb{C}$, where $\cdot$ is the scalar multiplication. The block matrix $\boldsymbol{P} \in \mathbb{C}^{D N \times N}$ is called invertible with $*$ operator if there exists $\boldsymbol{Q} \in \mathbb{C}^{D N \times N}$ such that

$$
\boldsymbol{P} * \boldsymbol{Q}=\boldsymbol{Q} * \boldsymbol{P}=\boldsymbol{I}_{D, N},
$$

where $\boldsymbol{I}_{D, N} \in \mathbb{C}^{D N \times N}$ and each block is defined as

$$
\left(\boldsymbol{I}_{D, N}\right)_{m, n}=\left\{\begin{array}{ll}
\mathbf{1}_{D} & \text { if } m=n \\
\mathbf{0}_{D} & \text { if } m \neq n
\end{array},\right.
$$

where $\mathbf{1}_{D} \in \mathbb{C}^{D}$ is the ones vector ${ }^{1}$ and $\mathbf{0}_{D} \in \mathbb{C}^{D}$ is the zeros vector ${ }^{2}$. Also, $\boldsymbol{I}_{D, N}=\bigvee_{d \in \mathbb{Z}_{D}} \boldsymbol{I}_{N}$ and $\boldsymbol{I}_{D, N} * \boldsymbol{C}=\boldsymbol{C}$, for all $\boldsymbol{C} \in \mathbb{C}^{D N \times P}$.

We denote $\boldsymbol{Q}=\boldsymbol{P}^{-1 *}$. The following new theorem explains conditions for what a block matrix is invertible in $\mathbb{C}^{D N \times N}$ with respect to $*$ operator.

Theorem 1. Let $\boldsymbol{P} \in \mathbb{C}^{D N \times N}$ and $\left\{\boldsymbol{P}_{d}\right\}_{d \in \mathbb{Z}_{D}} \subset \mathbb{C}^{N \times N}$ its generator set. $\boldsymbol{P}$ is invertible with $*$ operator if and only if each $\boldsymbol{P}_{d}$ is invertible. Also, $\boldsymbol{P}^{-1 *}$ is unique and

$$
\boldsymbol{P}^{-1 *}=\bigvee_{d \in \mathbb{Z}_{D}}\left(\boldsymbol{P}_{d}\right)^{-1}
$$

Proof. If $\boldsymbol{P}$ is invertible, then exists $\boldsymbol{Q} \in \mathbb{C}^{D N \times N}$ with generator set $\left\{\boldsymbol{Q}_{d}\right\}_{d \in \mathbb{Z}_{D}} \subset$ $\mathbb{C}^{N \times N}$, such that $\boldsymbol{P} * \boldsymbol{Q}=\boldsymbol{I}_{D, N}$. Then:

$$
\begin{aligned}
\boldsymbol{P} \text { is invertible } & \Leftrightarrow \boldsymbol{P} * \boldsymbol{Q}=\boldsymbol{I}_{D, N} \\
& \Leftrightarrow \bigvee_{d \in \mathbb{Z}_{D}} \boldsymbol{P}_{d} \boldsymbol{Q}_{d}=\bigvee_{d \in \mathbb{Z}_{D}} \boldsymbol{I}_{N} \\
& \Leftrightarrow \boldsymbol{P}_{d} \boldsymbol{Q}_{d}=\boldsymbol{I}_{N} \text {, for all } d \in \mathbb{Z}_{N} \\
& \Leftrightarrow \boldsymbol{Q}_{d}=\boldsymbol{P}_{d}^{-1}, \text { for all } d \in \mathbb{Z}_{N} \\
& \Leftrightarrow \boldsymbol{P}_{d} \text { is invertible, for all } d \in \mathbb{Z}_{N}
\end{aligned}
$$

This proves that $\left\{\boldsymbol{P}_{d}^{-1}\right\}_{d \in \mathbb{Z}_{D}} \subset \mathbb{C}^{N \times N}$ is the generator set of $\boldsymbol{P}^{-1 *}$. Also, generator set is indexed and unique, then $\boldsymbol{P}^{-1 *}$ is unique.

\section{Vector-Valued Discrete Fourier Transform}

Benedetto and Donatelli [2] define a kind of DFT by $N$-periodic signal, taking values in $\mathbb{C}^{D}$, with DFT frames as transform kernel. This type of DFT is called vector-valued discrete Fourier transform, or simply, vector-valued DFT. Signals for this transform are represented by a block vector in $x \in \mathbb{C}^{D N}$, where each block $x_{n}$ is a complex vector of size $D$. Let $\left\{1 / \sqrt{D} \cdot e_{N}^{n}\right\}_{n \in \mathbb{Z}_{N}}$ a DFT

\footnotetext{
${ }^{1}$ The ones vector is defined as $\mathbf{1}_{D}(n)=1$, for all $n \in \mathbb{Z}_{D}$

${ }^{2}$ The zeros vector is defined as $\mathbf{0}_{D}(n)=0$, for all $n \in \mathbb{Z}_{D}$
} 
frame for $\mathbb{C}^{D}$, with $\mathcal{A}=\left\{\alpha_{1}, \alpha_{2}, \ldots, \alpha_{D}\right\}$. The vector-valued DFT of $x$ is the $\operatorname{map} \mathcal{F}_{x}^{\mathcal{A}}: \mathbb{Z}_{N} \rightarrow \mathbb{C}^{D}$ such that

$$
\mathcal{F}_{x}^{\mathcal{A}}(k)=\frac{1}{\sqrt{D}} \sum_{n \in \mathbb{Z}_{N}} x_{n} \odot e_{N}^{-n k} .
$$

If $D=1$ and $\mathcal{A}=\{1\}$, then the vector-valued DFT is the classical DFT. The vector-valued DFT is not unique, because it depends of elements of set $\mathcal{A}=\left\{\alpha_{1}, \alpha_{2}, \ldots, \alpha_{D}\right\}$. Thus, each element in $\mathbb{C}^{D N}$ has $\frac{N !}{D !(N-D) !}$ different vectorvalued DFTs representations.

The vector-valued DFT can be represented as block matrix and it is defined as $\boldsymbol{F}_{N}^{\mathcal{A}} \in \mathbb{C}^{D N \times N}$, such that each block $(m, n)$ is equal to vector $1 / \sqrt{D} \cdot e_{N}^{-m n}$ and it is called vector-valued DFT block matrix. Also, the block matrix representation of vector-valued DFT of $x \in \mathbb{C}^{D N}$ is $\mathcal{F}_{x}^{\mathcal{A}}=\boldsymbol{F}_{N}^{\mathcal{A}} * x$. The set generator of $\boldsymbol{F}_{N}^{\mathcal{A}}$ is $\left\{1 / \sqrt{D} \cdot \bigodot_{m \in \mathbb{Z}_{\alpha_{d}}} \boldsymbol{F}_{N}\right\}_{d \in \mathbb{Z}_{D}}$, i.e.,

$$
\boldsymbol{F}_{N}^{\mathcal{A}}=\bigvee_{d \in \mathbb{Z}_{D}}\left(\frac{1}{\sqrt{D}} \bigodot_{m \in \mathbb{Z}_{\alpha_{d}}} \boldsymbol{F}_{N}\right)
$$

In the following new theorem, necessary and sufficient new conditions are presented in order to know when the vector-valued DFT block matrix is invertible with respect to $*$ operator.

Theorem 2. Let two positive integer numbers $N$ and $D, N \geq D$, and $\boldsymbol{F}_{N}^{\mathcal{A}} \in$ $\mathbb{C}^{D N \times N}$, with $\mathcal{A}=\left\{\alpha_{1}, \alpha_{2}, \ldots, \alpha_{D}\right\}$ :

1. if $\alpha_{1}=0$, then $\boldsymbol{F}_{N}^{\mathcal{A}}$ is not invertible with respect to $*$ operator.

2. if $N$ is prime and $\alpha_{1} \neq 0$, then $\boldsymbol{F}_{N}^{\mathcal{A}}$ is invertible with respect to $*$ operator.

3. if $N$ is composite, then:

(a) if g.c.d. $\left(N, \alpha_{d}\right)=1$, for all $d \in \mathbb{Z}_{D}$, then $\boldsymbol{F}_{N}^{\mathcal{A}}$ is invertible with respect to $*$ operator.

(b) if g.c.d.( $\left(N, \alpha_{d}\right) \neq 1$, for some $d \in \mathbb{Z}_{D}$, then $\boldsymbol{F}_{N}^{\mathcal{A}}$ is not invertible with respect to $*$ operator.

4. if it is well defined, $\left(\boldsymbol{F}_{N}^{\mathcal{A}}\right)^{-1 *} \in \mathbb{C}^{D N \times N}$ is represented by

$$
\left(\boldsymbol{F}_{N}^{\mathcal{A}}\right)^{-1 *}=\frac{D}{N} \overline{\boldsymbol{F}_{N}^{\mathcal{A}}}
$$

Proof. Let $\boldsymbol{G}_{\alpha_{d}}=\bigodot_{n \in \mathbb{Z}_{d}} \boldsymbol{F}_{N}$. The generator set of $\boldsymbol{F}_{N}^{\mathcal{A}}$ is $\left\{1 / \sqrt{D} \cdot \boldsymbol{G}_{\alpha_{d}}\right\}_{d \in \mathbb{Z}_{D}}$. By Theorem $1, \boldsymbol{F}_{N}^{\mathcal{A}}$ is invertible if and only if $\boldsymbol{G}_{\alpha_{d}}$ is invertible, for all $d \in \mathbb{Z}_{D}$, and if $\boldsymbol{G}_{d}$ is not invertible, for some $d \in \mathbb{Z}_{D}$, then $\boldsymbol{F}_{N}^{\mathcal{A}}$ is not invertible. 
1. $\alpha_{1}=\min \{\mathcal{A}\}$ and $0 \leq \alpha_{1}<\alpha_{2}<\ldots<\alpha_{D}<N$, then if $\alpha_{d}=0$ then $d=1$. Now, if $\alpha_{1}=0$, then $\boldsymbol{G}_{\alpha_{1}}=\boldsymbol{U}_{N}$. It is clear that $\boldsymbol{G}_{\alpha_{1}}$ is not invertible. Thus, it follows that $\boldsymbol{F}_{N}^{\mathcal{A}}$ is not invertible.

2. As $\alpha_{1} \neq 0$, then $0<\alpha_{d}<N$, for all $d \in \mathbb{Z}_{D}$. In addition, as $N$ is a prime number, then by Lemma $1, \boldsymbol{G}_{\alpha_{d}}$ is invertible for all $\alpha_{d}<N$. Thus, it follows that $\boldsymbol{F}_{N}^{\mathcal{A}}$ is invertible.

3. In this case, $N$ is a composite number.

(a) As explained above, $\boldsymbol{G}_{\alpha_{d}}(m, n)=\omega_{N}^{-m n \alpha_{d}}$, for $(m, n) \in \mathbb{Z}_{N} \times \mathbb{Z}_{N}$ and $\alpha_{d} \in \mathcal{A}$. Let $\boldsymbol{H}_{\alpha_{d}} \in \mathbb{C}^{N \times N}$ such that $\boldsymbol{H}_{\alpha_{d}}(m, n)=\frac{1}{N} \omega_{N}^{m n \alpha_{d}}$. Then

$$
\begin{aligned}
\boldsymbol{G}_{\alpha_{d}} \boldsymbol{H}_{\alpha_{d}}(m, n) & =\frac{1}{N} \sum_{k \in \mathbb{Z}_{N}} \omega_{N}^{-m k \alpha_{d}} \omega_{N}^{k n \alpha_{d}} \\
& =\frac{1}{N} \sum_{k \in \mathbb{Z}_{N}} \omega_{N}^{k \alpha_{d}(n-m)}
\end{aligned}
$$

If $m=n$, then $\boldsymbol{G}_{\alpha_{d}} \boldsymbol{H}_{\alpha_{d}}(m, n)=1$, by statement (2). But, if $m \neq n$, then

$$
n-m \in\{-(N-1), \ldots,-1,1, \ldots N-1\}
$$

By hypothesis, g.c.d. $\left(N, \alpha_{d}\right)=1$, for all $\alpha_{d} \in \mathcal{A}$. Then $(m-n) \alpha_{d} \not \equiv$ $0 \bmod N$. Thus, by statement $(2), \boldsymbol{G}_{\alpha_{d}} \boldsymbol{H}_{\alpha_{d}}(m, n)=0$. Then $\boldsymbol{G}_{\alpha_{d}} \boldsymbol{H}_{\alpha_{d}}=\boldsymbol{I}_{N}$ and $\boldsymbol{H}_{\alpha_{d}}=\left(\boldsymbol{G}_{\alpha_{d}}\right)^{-1}$ for all $\alpha_{d} \in \mathcal{A}$. Thus, each element of $\left\{1 / \sqrt{D} \cdot \boldsymbol{G}_{\alpha_{d}}\right\}_{d \in \mathbb{Z}_{D}}$ is invertible. It follows that $\boldsymbol{F}_{N}^{\mathcal{A}}$ is invertible.

(b) If g.c.d. $\left(N, \alpha_{d}\right) \neq 1$, then there are positive integers $k, m_{1}, m_{2} \in$ $\mathbb{Z}_{N} \backslash\{0,1\}$ such that $N=m_{1} k$ and $\alpha_{d}=m_{2} k$. Let $\boldsymbol{G}_{k}\left(m_{1},:\right)$ and $\boldsymbol{G}_{k}\left(m_{2},:\right)$ the rows $m_{1}$ and $m_{2}$ of $\boldsymbol{G}_{k}$, respectively. Then

$$
\boldsymbol{G}_{k}\left(m_{1}, n\right)=\omega_{N}^{-n m_{1} k}=\omega_{N}^{-n N}=1
$$

and

$$
\boldsymbol{G}_{k}\left(m_{2}, n\right)=\omega_{N}^{-n m_{2} k}=\omega_{N}^{-n N}=1,
$$

for all $n \in \mathbb{Z}_{N}$. Thus, the rows $m_{1}$ and $m_{2}$ are equals, thus $\boldsymbol{G}_{k}$ is not invertible. It follows that $\boldsymbol{F}_{N}^{\mathcal{A}}$ is not invertible. 
4. From equation (6), the set $\left\{\sqrt{D} / N \cdot \overline{\boldsymbol{G}_{\alpha_{d}}}\right\}_{d \in \mathbb{Z}_{D}}$ is the generator set of $\left(\boldsymbol{F}_{N}^{\mathcal{A}}\right)^{-1 *}$. Then

$$
\begin{aligned}
\left(\boldsymbol{F}_{N}^{\mathcal{A}}\right) *\left(\boldsymbol{F}_{N}^{\mathcal{A}}\right)^{-1 *} & =\bigvee_{d \in \mathbb{Z}_{D}}\left[\left(\frac{1}{\sqrt{D}} \boldsymbol{G}_{\alpha_{d}}\right)\left(\frac{\sqrt{D}}{N} \overline{\boldsymbol{G}_{\alpha_{d}}}\right)\right] \\
& =\bigvee_{d \in \mathbb{Z}_{D}} \boldsymbol{I}_{N} \\
& =\boldsymbol{I}_{D, N}
\end{aligned}
$$

Corollary 1. Let two positive integer numbers $N$ and $D, N \geq D$, and $\boldsymbol{F}_{N}^{\mathcal{A}} \in$ $\mathbb{C}^{D N \times N}$, with $\mathcal{A}=\{1,2, \ldots, D\}$. If $N$ is a composite number and $r \in \mathbb{Z}_{N} \backslash\{0\}$ is the smallest prime number such that divide $N$, then:

1. if $D<r$, then $\boldsymbol{F}_{N}^{\mathcal{A}}$ is invertible with respect to $*$ operator.

2. if $D \geq r$, then $\boldsymbol{F}_{N}^{\mathcal{A}}$ is not invertible with respect to $*$ operator.

Proof.

1. $r$ is the smallest prime number such that $r$ divide $N$ and $D<r$, then g.c.d. $(N, D)=1$. Thus, $\boldsymbol{F}_{N}^{\mathcal{A}}$ is invertible, by Theorem 2 .

2. $r$ is the smallest prime number such that $r$ divide $N$ and $D \geq r$, then g.c.d. $(N, D) \neq 1$. Thus, $\boldsymbol{F}_{N}^{\mathcal{A}}$ is not invertible, by Theorem 2 .

From Theorem 2, a new vector-valued DFT inversion is defined. The following corollary presents necessary and sufficient conditions to vector-valued DFT inversion, and so, it makes a perfect recovery of signal $x$.

Corollary 2. Let $x \in \mathbb{C}^{D N}$ and $\left\{1 / \sqrt{D} \cdot e_{N}^{n}\right\}_{n \in \mathbb{Z}_{N}}$ a DFT frames for $\mathbb{C}^{D}$ with $\mathcal{A}=\left\{\alpha_{1}, \alpha_{2}, \ldots, \alpha_{D}\right\}$, such that $\alpha_{1} \neq 0$. If $N$ is a prime or $N$ is a composite such that g.c.d. $\left(N, \alpha_{d}\right)=1$, for all $d \in \mathbb{Z}_{N}$, then the vector-valued DFT inversion is well-defined and it is defined as $\left(\mathcal{F}_{x}^{\mathcal{A}}\right)^{-1}: \mathbb{Z}_{N} \rightarrow \mathbb{C}^{D}$ such that

$$
\left(\mathcal{F}_{x}^{\mathcal{A}}\right)^{-1}(n)=x_{n}=\frac{\sqrt{D}}{N} \sum_{k \in \mathbb{Z}_{N}} \mathcal{F}_{x}^{\mathcal{A}}(k) \odot e_{N}^{n k}
$$

Otherwise, the vector-valued DFT inversion does not exist. 
Proof. The block matrix representation of $\mathcal{F}_{x}^{\mathcal{A}}$ is $\boldsymbol{F}_{N}^{\mathcal{A}} * x$. From Theorem 2, the inverse of $\boldsymbol{F}_{N}^{\mathcal{A}}$ exists. Then

$$
\begin{aligned}
\left(\boldsymbol{F}_{N}^{\mathcal{A}}\right)^{-1 *} *\left(\boldsymbol{F}_{N}^{\mathcal{A}} * x\right) & =\left(\left(\boldsymbol{F}_{N}^{\mathcal{A}}\right)^{-1 *} * \boldsymbol{F}_{N}^{\mathcal{A}}\right) * x \\
& =\boldsymbol{I}_{D, N} * x \\
& =x
\end{aligned}
$$

Besides, algebraic representation of $\left(\boldsymbol{F}_{N}^{\mathcal{A}}\right)^{-1 *} *\left(\boldsymbol{F}_{N}^{\mathcal{A}} * x\right)$ is expressed in equation (7).

\section{Conclusions}

This paper presented a study of the vector-valued DFT and your inversion transform. In particular, we presents necessary and sufficient new conditions for vector-valued DFT inversion. Certain conditions on the elements of $\mathcal{A}$ were imposed, and so, it can make a perfect recovery of any signal. This study was performed through the block matrix representation of vector-valued DFT. We present necessary and sufficient new conditions to determine when the vector-valued DFT block matrix is invertible. In addition, we explained and developed some new concepts related to the block matrix space.

Acknowledgments. This research was supported by Instituto Tecnológico de Costa Rica through Vicerrectoría de Investigación y Extensión.

\section{References}

[1] J.J. Benedetto and J.J. Donatelli. Ambiguity function and frame-theoretic properties of periodic zero-autocorrelation waveforms. IEEE Journal of Selected Topics in Signal Processing, 1(1):6-20, june 2007.

[2] J.J. Benedetto and J.J. Donatelli. Frames and a vector-valued ambiguity function. In 42nd Asilomar Conference on Signals, Systems and Computers, pages 8-12, oct. 2008.

[3] John J. Benedetto and Travis D. Andrews. Intrinsic wavelet and frame applications. In Proc. SPIE 8058, may 2011.

[4] L. Berman and Feuer A. On perfect conditioning of vandermonde matrices on the unit circle. The Electronic Journal of Linear Algebra, 16:157-161, july 2007 . 
[5] W. Khan, I.M. Qureshi, and K. Sultan. Ambiguity function of phased mimo radar with colocated antennas and its properties. IEEE Geoscience and Remote Sensing Letters, 11(7):1220-1224, July 2014.

[6] M.S. Richman, T.W. Parks, and R.G. Shenoy. Discrete-time, discretefrequency, time-frequency analysis. IEEE Transactions on Signal Processing, 46(6):1517-1527, Jun 1998.

[7] S.S. Soliman and R.A. Scholtz. Spread ambiguity functions. IEEE Transactions on Information Theory, 34(2):343-347, Mar 1988.

[8] P. Stinco, M.S. Greco, F. Gini, and M. Rangaswamy. Ambiguity function and cramer-rao bounds for universal mobile telecommunications systembased passive coherent location systems. IET Radar, Sonar Navigation, 6(7):668-678, August 2012.

[9] F. Zhang. In Matrix Theory, Universitext. Springer New York, 2011.

Received: February 26, 2015; Published: March 27, 2015 